adherence to the guidelines: it is fundamental continuing the training of the staff to achieve the required standard. Among the objectives for 2013, another audit with a modified cheque list will be performed, involving a greater number of health care professionals.

No conflict of interest.

\section{GRP-026 APPLICATION OF FAILURE MODE AND EFFECT ANALYSIS ON THE PRESCRIBING AND TRANSCRIBING PROCESSES IN THE DISTRIBUTION UNIT DOSE SYSTEM}

\author{
doi:10.1136/ejhpharm-2013-000276.026
}

E Molina, H Mateo, S Cifuentes, P Rodriguez, P Nieto, JJ Fernandez, I Alferez. Hospital Torrecárdenas, Servicio de Farmacia, Almeria, Spain

Background Failure Mode and Effect Analysis (FMEA) is a tool to identify, assess and prevent possible failures that could occur in a process.

Purpose

1. To describe FMEA as a method to identify weaknesses in the process of prescription and transcription of medical orders.

2. To isolate the key steps according to their risk priority number (Rpn).

3. To report the steps taken.

Materials and Methods A multidisciplinary study group was assembled. Possible errors in the prescription/transcription workflow were identified and classified according to their RPN score (calculated by multiplying the severity, occurrence, and detection). Strategies for improvement were established.

Results Errors in the prescription were classified as follows: (1) Patient-and-history identification, (2) Clinical and laboratory data checkout, (3) Treatment conciliation, (4) Allergies, (5) Verbal prescription, (6) Handwritten prescription. Errors in transcription: (7) Patient identification (nurse), (8) Internally mailed prescriptions, (9) Paper transcription, (10) Check in pharmacy, (11) Patient identification (pharmacist), (12) Prescription validation, (13) Prescription printing, and (14) Acknowledgement of errors by the pharmacist. Top-ranked item (number), suggested solution, and indicator, respectively were: (5) Verbal prescription (288), storage of verbal prescriptions in pharmacy, \% of verbal prescriptions; (9) Failure in paper transcription (288), computerised physician order entry (CPOE), \% of electronic prescriptions; (14) Error report to the pharmacist (288), implementation of a two-way communication protocol, number of reports; (8) Paper-based prescriptions sent to pharmacy (243), CPOE, \% of electronic prescriptions; (10) Check in pharmacy (216), CPOE, \% of electronic prescriptions. The pharmacy, medical director, and Quality Unit were responsible for the changes undertaken in all cases.

Conclusions Verbal prescription, failure in paper transcription, error report and mailed prescriptions to pharmacy were the steps with the highest risk of error. For most cases, CPOE was implemented, whereas the percentage of electronic prescriptions was the key indicator to measure the overall improvement in these processes. In conclusion, further efforts and pharmacy policies should focus on the implementation of CPOE in all inpatient areas, thus preventing failure of prescription/transcription and validation loops.

No conflict of interest.

\section{GRP-027 ASSESSMENT OF BLOOD PRESSURE CONTROL AND ANTIHYPERTENSIVE MEDICATION ADHERENCE IN A PORTUGUESE HYPERTENSIVE POPULATION}

doi:10.1136/ejhpharm-2013-000276.027

${ }^{1} \mathrm{M}$ Morgado, ${ }^{2} \mathrm{~J}$ Silva, ${ }^{2} \mathrm{~A}$ Almeida. ${ }^{1}$ Hospital Centre of Cova da Beira, Pharmaceutical Services, Covilhã, Portugal; '2University of Beira Interior, Health Sciences Faculty, Covilhã, Portugal
Background Hypertension is one of the major causes of worldwide morbidity and mortality. Despite the wide variety and availability of powerful antihypertensive agents, the blood pressure (BP) of fewer than a third of adult hypertensive patients is under control. Non-adherence to medicines is one of the major causes of treatment failure.

Purpose To evaluate BP control and antihypertensive medicines adherence in a Portuguese hypertensive population.

Materials and Methods A cross-sectional observational study was conducted in adult (aged 18 or over) hypertensive patients attending the hypertension/dyslipidaemia clinic for at least 6 months at the university teaching hospital of Cova da Beira Hospital Centre, Covilhã, Portugal, from March to August 2012. Patients were asked to participate in a structured interview which included socio-demographic characteristics, antihypertensive medicines adherence and target BP values. Medicines adherence was measured using a validated five-item adherence scale, [1] derived from the four-item scale developed by Morisky et al, [2] Detailed clinical information was obtained from medical records.

Results A total of 94 patients met the inclusion criteria and completed the structured interview. Of these, the BP of $47 \%$ was under control according to the European Society of Hypertension. Antihypertensive medicines adherence was $40 \%$. Patients with controlled BP had a significantly higher rate of medicines adherence than patients with uncontrolled BP ( $52 \%$ vs. $30 \%, \mathrm{P}=0.028)$. Likewise, it was observed that patients whose BP was controlled were significantly more aware of their target BP figures ( $75 \%$ vs. $46 \%, \mathrm{P}=0.034)$. Conclusions Many hypertensive patients prescribed antihypertensive treatment fail to achieve BP control in clinical practise. Poor medicines adherence and poor patient knowledge of target BP values should be considered as possible underlying causes of inadequately controlled BP and must be addressed in any intervention aimed to improve BP control.

\section{References}

1. Shea S, Misra D, Ehrlich MH, Field L, Francis CK. Correlates of nonadherence to hypertension measurement in an inner-city population. Am J Public Health 1992;82(12):1607-12.

2. Morisky DE, Green LW, Levine DM. Concurrent and predictive validity of a self-report measure of medication adherence. Med Care 1986;24(1):67-74.

No conflict of interest.

\section{GRP-028 ASSESSMENT OF COMPLIANCE AND AVOIDED COSTS AFTER IMPLEMENTATION OF GUIDELINES FOR CANDIDA INFECTION TREATMENT AND INVASIVE FUNGAL INFECTIONS IN NON-HAEMATOLOGY PATIENTS}

doi:10.1136/ejhpharm-2013-000276.028

GJ Nazco Casariego, M Bullejos Molina, S Duque Fernandez, N Yurrebaso Eguior, I Gonzalez Perera, F Gutierrez Nicolas, J Gonzalez, M Chafer Rudilla. Hospital Universitario de Canarias, Pharmacy, La Laguna, Spain

Background The recent marketing of new high-cost antifungal agents (echinocandins and azoles) requires the design of costeffective treatment protocols.

Purpose A new treatment guide for candidaemia and other invasive fungal infections for non-haematology adult patients was approved in June 2011. The main objective was to evaluate the cost reduction by introducing this protocol in a 737-bed University Hospital serving a population of more than 400,000 inhabitants.

Materials and Methods Retrospective observational study between June and December 2011. We reviewed the medical records of patients whom were prescribed antifungal treatment during that time and we assessed the adjustment to the approved treatment guidelines. To quantify the avoided costs we extracted consumption data and costs of antifungals from the pharmacy service 
management system $\left(\mathrm{SAP}^{\circledR}\right)$ and compared them with the same period the previous year.

Results During the study 43 non-haematology patients were treated with antifungal agents. In 38 patients (88.4\%) the approved treatment guidelines were followed and in 5 patients $(11.6 \%)$ they were breached.The most significant breaches occurred in internal medicine $(22.2 \%)$ and in critical care $(3.7 \%)$.

Regarding avoided costs for the six months of the study, antifungal costs were reduced by 240,616 euros. We observed a $61.9 \%$ and $48 \%$ increase in use in fluconazole and anidulafungin, and a $42.8 \%$ and $41.7 \%$ decrease in caspofungin and liposomal amphotericin B use. These results are consistent with the recommendations contained in the guide (first line use of fluconazole in nonimmunosuppressed patients and in azole resistance use anidulafungin). Micafungin use was restricted to the paediatric population with consumption equal to that in the previous period.

Conclusions The treatment guideline compliance was excellent at our hospital, resulting in a significant decrease in antifungal expenses. Implementation of these guidelines in the management of high-cost drugs resulted in significant cost reductions and therefore in a more rational use of healthcare budgets.

No conflict of interest.

\section{GRP-029 ASSESSMENT OF THE THERAPEUTIC MANAGEMENT OF PATIENTS ON WEEKEND LEAVE}

doi:10.1136/ejhpharm-2013-000276.029

${ }^{1}$ A Camerlynck, 'S Allemon-Dewulf, $2 \mathrm{~V}$ Herlin, 'M Delobel. 'HOPITAL MARITIME, Pharmacy, Zuydcoote, France; ${ }^{2}$ HOPITAL MARITIME, Quality department, Zuydcoote, France

Background A patient's suicide attempt with benzodiazepines was reported to our quality department. The patient ingested a bottle of drops given for his weekend leave. According to French regulations, patients are allowed to leave hospital for at most 48 hours but administratively they are still hospitalised and under the director's responsibility. Their medicines must be provided for this period.

Purpose To assess the therapeutic management of patients on weekend leave in order to highlight opportunities for improvement. Materials and Methods We performed an audit of the medical management of patients on weekend leave. The audit was performed using a form containing open questions. One nurse from each department was audited.

Results Although nurses can't refer to any procedures on this topic, all care units provide medicines by strictly following the prescription. Multidose vials (drops, syrups, etc.) are not unpacked and are given in their entirety. One care unit out of nine mentioned that patients are stated to be on leave in the patient's medical record. Only $22 \%$ of audited nurses systematically put the treatment in a pillbox. Several nurses reported that pillboxes weren't available which resulted in treatments being bulk packed in a bag by $66 \%$ of wards or in a plastic pot by $11 \% .56 \%$ of treatments were delivered with the care plan coming from the patient record.

Conclusions The audit highlighted the need to standardise practises (traceability, packaging of treatment and the presence of a care plan) and improve safety, to purchase daily pillboxes for all wards and to solve technical problems for delivering multidose medicines.

The pharmacy, in cooperation with the quality department, wrote a procedure in order to refocus the medical management of patients going on leave. The pharmacy is now responsible for delivering oral syringes for drinkable solutions in order to prevent such an accident from happening again, by delivering the exact amount prescribed.

No conflict of interest.

\section{GRP-030 AUDIT OF PHARMACISTS' INTERVENTIONS WHEN SCREENING ADULT CHEMOTHERAPY PRESCRIPTIONS ON AN ELECTRONIC PRESCRIBING SYSTEM}

doi:10.1136/ejhpharm-2013-000276.030

${ }^{1} \mathrm{~N}$ Stoner, ${ }^{2} \mathrm{~S}$ Dhaliwal, ${ }^{2} \mathrm{C}$ Langran, ${ }^{1} \mathrm{E}$ Chan. ${ }^{1}$ Oxford University Hospitals NHS Trust, Pharmacy, Oxford, UK; ${ }^{2}$ The University of Reading, School of Pharmacy, Reading, UK

Background The UK Cancer Standards require there to be protocols for chemotherapy treatment. The Thames Valley Cancer Network (TVCN) has developed and maintained network-wide protocols, which are continually updated for each tumour sitespecific group. Clinical verification of chemotherapy prescriptions by pharmacists is an essential step to ensure patient safety and compliance with protocols, in line with national standards.

Purpose To audit pharmacists' interventions when clinically screening adult chemotherapy, clinical trial and supportive care prescriptions for oncology and haematology patients in the Oxford University Hospitals NHS Trust (OUH), and to ascertain the level of compliance of these prescriptions with relevant protocols and guidelines. To compare results with the audit undertaken in OUH in 2010. Materials and Methods Pharmacists clinically screening the prescriptions completed an intervention form at the time of screening to enable prospective data collection over a three-week period. The screening pharmacists graded the intervention at the time of data collection, and interventions were subsequently independently graded by the investigator. The results of this audit are compared to a previous audit carried out for $\mathrm{OUH}$, and the aim was to compare interventions during the two audit years.

Results The OUH had a decrease in the number of interventions made by $24 \%$ compared to the audit in 2010 . The number of moderate and major interventions made also decreased by $5 \%$ and $23 \%$ respectively. Time spent on making interventions also decreased. Incorrect frequency/duration/date of treatment, inappropriate dose, confirmation of dose/regimen/prescriptions were prescribed according to guidelines compared to just $68 \%$ in 2010 .

Conclusions The changes implemented after the OUH audit in 2010 were successful and this is seen in the results. To improve further this audit should be conducted across TVCN hospitals every year so that each hospital can monitor their progress. Having regular training days for clinicians would be beneficial.

No conflict of interest.

\section{GRP-031 BARCODE TECHNOLOGY ON THE SAFETY OF CYTOSTATIC DRUGS ADMINISTRATION, ONE YEAR EVALUATION}

doi:10.1136/ejhpharm-2013-000276.031

MC Serrano Vicente, A Martínez Crespo, MC Viñuales Armengol, MP Amador Rodríguez, M Cabrero Ciprés. Hospital San Jorge, Pharmacy, Huesca, Spain

Background Technology has been developed to verify medicines by incorporating barcode verification technology within an electronic medicines-administration system (eMAR barcode) to prevent serious medicines errors during administration of medicines.

Purpose To evaluate the implementation of an electronic system of validation and control of cytostatic drug administration using barcodes and an electronic medicines-administration system (eMAR).

Materials and Methods To identify patients we used barcoded wristbands and we acquired PDAs as eMAR, which were connected to the e-prescribing programme by the hospital WIFI.

After having received the medicine sent from the Pharmacy Department, the nurse scans the barcodes printed on the patient's wristband, then drug information about the medicines to be administered appears on the screen of the PDA (patient data, route, speed and time of administration, sequence order, components, and number of administrations). After scanning the barcode on the patient's 